\title{
Erratum zu: EU-Professionalismus als transnationales Feld
}

\author{
Sebastian M. Büttner • Steffen Mau
}

Online publiziert: 1. August 2014

(C) Springer Fachmedien Wiesbaden 2014

\section{Erratum zu: \\ Berlin J Soziol (2014) 24:141-167 \\ DOI 10.1007/s11609-014-0246-7}

Im ersten Absatz des Abschnitts 4 wurde im Zuge der Publikation versehentlich eine sinnentstellende Textpassage eingefügt. Statt:

\section{Vom bürokratischen Kern zum europäischen Feld: Das transnationale Feld des EU-Professionalismus}

Wir können an dieser Stelle keine umfassende Beschreibung der Emergenz des Feldes des EU-Professionalismus liefern. Die eingehende Beschreibung einzelner Tätigkeiten und Tätigkeitsbereiche kann letztlich nur durch viele einzelne empirische Studien und Detailanalysen erfolgen (siehe dazu etwa die Sammelbände von Georgakakis und Rowell 2013 oder von Vauchez und de Witte 2013). Das Ziel der nachfolgenden Darstellung ist es, die Professionalisierung von EU-Expertise feldtheoretisch zu rahmen und damit zu zeigen, inwiefern Professionalisierung selbst Teil der Feldgenese ist und als spezifische Handlungsstrategie von Akteuren zum Tragen

The Onlineversion des Originalbeitrages ist erreichbar unter doi: 10.1007/s11609-014-0246-7

\footnotetext{
S. M. Büttner $(\bowtie)$

Institut für Soziologie, Friedrich-Alexander-Universität Erlangen-Nürnberg,

Kochstr. 4, 91054 Erlangen, Deutschland

E-Mail: sebastian.buettner@fau.de

S. Mau

Bremen International Graduate School of Social Sciences (BIGSSS),

Postfach 3304 40, 28334 Bremen, Deutschland

E-Mail: smau@bigsss.uni-bremen.de
} 
kommt. Wie bereits angedeutet, gehen wir nicht von der Existenz eines einheitlichen, fest etablierten Feldes von EU-spezifischen Tätigkeiten aus, sondern von der Herausbildung eines "schwachen Feldes“ (Vauchez 2008), das nicht auf einheitlichen, fest umgrenzten Feldern, nicht zuletzt durch die Ex Regeln und Ressourcen beruht, sondern intern stark differenziert und fragmentiert ist. Anders als etwa im Fall der klassischen Ausbildung von etablierten „Professionen“ oder von Verwaltungsbeamten in nationalen Bürokratien werden nicht alle Mitglieder des Feldes durch einheitliche und fest etablierte Ausbildungsgänge und Karriereverläufe in das Feld hineinsozialisiert. Auch ist die Entwicklung des Feldes stark mit Entwicklungen in umliegenden Feldern verknüpft - etwa im Feld der Europapolitik und in den einzelnen nationalen Politikfeldern - oder mit Entwicklungen in anderen professionellen Feldern, nicht zuletzt durch die Expansion der Managementberatung im Feld des Managements (siehe dazu den Beitrag von Schmidt-Wellenburg in diesem Heft).

soll dieser korrekt wie folgt lauten:

\section{Vom bürokratischen Kern zum europäischen Feld: Das transnationale Feld des EU-Professionalismus}

Wir können an dieser Stelle keine umfassende Beschreibung der Emergenz des Feldes des EU-Professionalismus liefern. Die eingehende Beschreibung einzelner Tätigkeiten und Tätigkeitsbereiche kann letztlich nur durch viele einzelne empirische Studien und Detailanalysen erfolgen (siehe dazu etwa die Sammelbände von Georgakakis und Rowell 2013 oder von Vauchez und de Witte 2013). Das Ziel der nachfolgenden Darstellung ist es, die Professionalisierung von EU-Expertise feldtheoretisch zu rahmen und damit zu zeigen, inwiefern Professionalisierung selbst Teil der Feldgenese ist und als spezifische Handlungsstrategie von Akteuren zum Tragen kommt. Wie bereits angedeutet, gehen wir nicht von der Existenz eines einheitlichen, fest etablierten Feldes von EU-spezifischen Tätigkeiten aus, sondern von der Herausbildung eines „schwachen Feldes“ (Vauchez 2008), das nicht auf einheitlichen, fest umgrenzten Regeln und Ressourcen beruht, sondern intern stark differenziert und fragmentiert ist. Anders als etwa im Fall der klassischen Ausbildung von etablierten „Professionen“ oder von Verwaltungsbeamten in nationalen Bürokratien werden nicht alle Mitglieder des Feldes durch einheitliche und fest etablierte Ausbildungsgänge und Karriereverläufe in das Feld hineinsozialisiert. Auch ist die Entwicklung des Feldes stark mit Entwicklungen in umliegenden Feldern verknüpft - etwa im Feld der Europapolitik und in den einzelnen nationalen Politikfeldern - oder mit Entwicklungen in anderen professionellen Feldern, nicht zuletzt durch die Expansion der Managementberatung im Feld des Managements (siehe dazu den Beitrag von Schmidt-Wellenburg in diesem Heft).

Der Verlag bittet, diesen Fehler zu entschuldigen. 